\title{
Down-regulation of miR-155 after treatment with narrow-band UVB and methotrexate associates with apoptosis of keratinocytes in psoriasis
}

Wipasiri Soonthornchai, ${ }_{1}^{1}$ Pattarin Tangtanatakul, ${ }^{1}$ Jitlada Meephansan, ${ }^{2}$ Kriangsak Ruchusatsawat, ${ }^{3}$ Rangsima Reantragoon, ${ }^{1}$ Nattiya Hirankarn, ${ }^{1}$ Jongkonnee Wongpiyabovorn ${ }^{1}$

\begin{abstract}
Background: Psoriasis is a chronic inflammatory skin disease arising from a complex interaction between genetics, epigenetics, the host's immune system and the environment. Recent accumulated data revealed the dysregulation of various microRNAs (miRNAs) in several diseases including psoriasis.
\end{abstract}

Objective: We explored the functional role and regulation of hsa-miR-155-5p (miR-155) in an immortalized keratinocyte cell line $(\mathrm{HaCaT})$, in relation to the pathogenesis and treatment of psoriasis.

Methods: miR-155 expression in normal skin and psoriatic skin lesion before and after treatment with methotrexate (MTX) and narrow-band ultraviolet B phototherapy (NB-UVB) were analyzed using quantitative reverse transcription PCR (qRT-PCR). Apoptotic activity, cell cycle and viable cells of miR-155 transfected HaCaT were measured using flow cytometry and MTS assay. Since, caspase-3 (CASP3) gene was predicted as a target gene of miR-155, the expression of CASP3 was detected in transfected $\mathrm{HaCaT}$ using western blot.

Results: We discovered that both MTX and NB-UVB significantly down-regulated miR-155 expression in psoriatic skin lesions. We also found that overexpression of miR-155 in HaCaT led to suppression of cell apoptosis and induced cell arrest at G0/G1 phase. Moreover, CASP3 expression was down-regulated in miR-155 transfected HaCaT.

Conclusion: This study demonstrates down-regulation of miR155 after treatment with MTX and NB-UVB in psoriatic skin lesion. miR155 plays significant role in apoptosis on HaCaT via CASP3. This finding provides a better understanding of the pathogenesis of psoriasis and might aid on developing the new monitoring tool or therapy for psoriasis in the future.

Key words: psoriasis, miR-155, methotrexate, NB-UVB, apoptosis

\footnotetext{
Affiliations:

${ }^{1}$ Center of Excellence in Immunology and Immune Mediated Diseases, Department of Microbiology, Faculty of Medicine, Chulalongkorn University, Bangkok, Thailand

2 Division of Dermatology, Chulabhorn International College of Medicine, Thammasat University, Pathum Thani, Thailand

National Institute of Health, Department of Medical Sciences, Nontaburi, Thailand
}

\section{Corresponding author:}

Jongkonnee Wongpiyabovorn

Division of Immunology, Department of Microbiology

Faculty of Medicine Chulalongkorn University

Rama 4 Road Bangkok 10330 Thailand

E-mail: jongkonnee.w@chula.ac.th, jongkonneew@gmail.com

\section{Introduction}

Psoriasis is a cell-mediated, chronic inflammatory skin disease, affecting approximately $2-3 \%$ of worldwide population. ${ }^{1}$ This disease has a significant effect on the patients' quality of life. ${ }^{1}$ The characteristic skin lesions include raised, well-demarcated, erythematous plaque covering with silvery scale. ${ }^{1}$ The pathogenesis of psoriasis is a complex interaction between genetics, epigenetics, the host's immune system and environment, leading to hyperproliferation and reducing in terminally differential keratinocytes with inflammatory cell infiltration in both the epidermis and dermis. ${ }^{1,2}$ In recent year, although several new biologic agents have been developed, methotrexate (MTX) and narrow-band ultraviolet B phototherapy (NB-UVB) are still frequently used as an effective and economical therapy for moderate to severe psoriasis, 
both MTX and NB-UVB influence on apoptosis and proliferation of keratinocytes. ${ }^{3,4}$ However, the precise mechanism that explains the efficiency of MTX and NB-UVB in psoriasis, in particular genetic regulation, still needs to be elucidated.

MIcroRNAs (miRNAs) are a novel class of highly conserved small non-coding RNAs containing about 21-25 nucleotides in length. ${ }^{5}$ They function as negative regulators of gene expression by mediating translational repression or degradation of target mRNAs, mainly found in the 3'-untranslated regions (UTR). ${ }^{6}$ miRNAs play crucial roles in several biological processes, i.e. proliferation, differentiation, apoptosis, signal transduction and organ development. ${ }^{6}$ Thus, the alteration of miRNA expression is associated with numerous diseases such as cancer, autoimmune diseases and auto-inflammatory diseases including psoriasis. ${ }^{7}$ Recent studies reveal that alteration of several miRNAs are associated with psoriasis. For example, miR-31, miR-203, miR-146a and miR-155 were intensively up-regulated, ${ }^{8-11}$ while miR-99a, miR-125b, miR194 and miR-217 were markedly down-regulated in psoriatic skin lesions. ${ }^{12-15}$ These miRNAs play roles in the pathogenesis and progression of psoriasis by various pathway including inflammation, keratinocyte apoptosis, proliferation and differentiation. Furthermore, these molecules are novel potential biomarkers for diagnosis, prognosis and therapeutic tools in psoriasis and other diseases. ${ }^{16}$

miR-155 has been shown to have various functions involving in inflammatory and immune response. ${ }^{17,18}$ Its expression plays an essential regulation in both innate and adaptive immune responses related to several diseases. Many studies showed the important functions of miR-155 in many immune cell types. ${ }^{17,18}$ For instance, miR-155 plays an important role in T-helper (Th) differentiation and maintenance of Th1, Th17 and macrophage inflammation by secreting their pro-inflammatory cytokines. ${ }^{17,22,23}$ In B cells, miR-155 acts as an antibody-mediated signaling regulator. ${ }^{19,20}$ Additionally, miR-155 serves as an oncogenic human miRNA which function as a key regulator of hematopoiesis and B-cell differentiation. ${ }^{20}$ To date, the functional role of miR-155 in keratinocytes related to psoriasis has not been clearly defined.

In this study, we hypothesized that aberrant levels of miR155 may play a critical role in immunopathogenesis of psoriasis, especially in dysfunction of keratinocyte. Therefore, the expression of miR-155 in normal skin from healthy individuals was compared to psoriatic skin lesions before and after treatment with MTX and NB-UVB. We also overexpressed mature miR-155 mimic in an immortalized keratinocyte cell lines $(\mathrm{HaCaT})$ to examine the influence of miR-155 on cell apoptosis, cell cycle and cell proliferation. Moreover, we identified the possible target gene of miR-155 that plays a role in reducing apoptotic activity in $\mathrm{HaCaT}$, which will lead to a better understanding of the functional roles and mechanism of regulation of miR-155. This knowledge would help to describe the critical roles of miR-155 in pathogenesis of psoriasis and may initiate the development of new effective therapeutic strategies in the future.

\section{Materials and methods Clinical specimens}

Eleven patients diagnosed with moderate to severe chronic plaque type psoriasis at King Chulalongkorn Memorial Hospital and 5 normal subjects were enrolled in the study. The severity of psoriasis was classified according to the Psoriasis Area and Severity Index (PASI score $\geq 10=$ moderate to severe) Seven patients were orally treated with $15 \mathrm{mg}$ MTX once a week and 4 patients were treated with NB-UVB irradiation three sessions per week for up to 12 weeks as a monotherapy. Patients with psoriatic arthritis, other autoimmune diseases, cancer, liver or renal disease were excluded from the study. All patients were omitted from all systemic therapies and photo-therapies for at least four weeks, and topical anti-psoriatic therapies for at least 2 weeks before specimen collection. Normal skin from normal subjects and lesional skin from patients with psoriasis at trunk were obtained before and after 4-week monotherapy with either MTX or NB-UVB. This research was approved by Research Affairs, Faculty of Medicine, Chulalongkorn University. Informed written consents were obtained from psoriasis patients and healthy individuals before enrolling in the study.

\section{miRNA and mRNA expression}

To examine the miRNA expression, the miRNAs were extracted from whole-tissue samples or cultured cells using the miRNeasy Mini Kit (Qiagen) according to the manufacturer's instructions for miRNA enrichment protocol to obtain small RNAs up to 200 bp. RT-qPCR was performed with TaqMan miRNA assays (Life Technologies), according to the manufacturer's instructions. Briefly, $10 \mathrm{ng}$ of miRNA fraction was reverse-transcribed in $15 \mu \mathrm{l}$ reaction with the TaqMan MicroRNA Reverse Transcription Kit (Life Technologies), and RT-qPCR was performed using TaqMan ${ }^{\circledR}$ Universal PCR Master Mix (Life Technologies). $1.33 \mu \mathrm{l}$ of cDNA was added to triplicate in $20 \mu \mathrm{l}$ PCR reactions. PCR was performed on a $7500 \mathrm{HT}$ thermocycler (Life Technologies). The expression of miR-155 was measured using the TaqMan primer/probe sets for miR-155 (assay ID 002623), and was normalized to the hsa-let-7a (assay ID 000377) for human tissue or RNU44 (assay ID 001094) for HaCaT keratinocyte. Relative gene expression levels were calculated according to the $2^{-\Delta \Delta \mathrm{Ct}}$ method. ${ }^{21}$

For studying mRNA expression, total RNA was extracted using TRIzol ${ }^{\mathrm{TM}}$ Reagent (Ambion) in accordance with the manufacturer's instructions. mRNA was performed with TaqMan Reverse Transcription Reagent (Life Technologies) in accordance with the manufacturer's instructions. Briefly, $500 \mathrm{ng}$ of total RNA was reverse-transcribed in $20 \mu \mathrm{l}$ reaction with the TaqMan Reverse Transcription Reagent (Life Technologies), and $500 \mathrm{ng}$ of cDNA was added to $10 \mu \mathrm{l}$ RT-qP$\mathrm{CR}$ reactions. All experiments were performed in duplicate. RT-qPCR amplification was conducted on a 7500 HT thermocycler (Life Technologies). The mRNA expression of CASP3 was normalized with GAPDH. Primer sequences were as follows: CASP3f: 5' GGA ATG ACA TCT CGG TCT GG 3' and CASP3r: 5' GGC TCA GAA GCA CAC AAA CA 3', GAPDHf: 5' ACC CAC TCC TCC ACC TTT 3' and GAPDHr: 5' CAC CAC CCT GTT GCT GTA G 3'. Relative gene expression levels were calculated according to the $2^{-\Delta \Delta \mathrm{Ct}}$ method. ${ }^{21}$ 


\section{HaCaT cells culture}

$\mathrm{HaCaT}$ was maintained in Dulbecco's Modified Eagle Medium (DMEM; Gibco, 11995), supplemented with 10\% fetal bovine serum (Gibco, 10270), 0.01 M HEPES (Gibco, 15630) and $100 \mathrm{U} / \mathrm{ml}$ penicillin-streptomycin (Gibco, 15140) and was incubated at $37^{\circ} \mathrm{C}$ in humidified incubator containing $5 \%$ $\mathrm{CO}_{2}$.

\section{Transient transfection}

$\mathrm{HaCaT}$ was seeded at $3 \times 10^{4}$ cells per well in a 24 -well plate or $6 \times 10^{4}$ cells per well in a 12 -well plate overnight and were transiently transfected with 50 or $100 \mathrm{nM}$ mirVana ${ }^{\infty}$ miRNA hsa-miR-155-5p mimic (4464066) or mirVana ${ }^{\circledR}$ miRNA negative control mimic (4464058). Transient transfections were performed using Lipofectamine RNAi Max Reagent according to the manufacturer's instructions (Invitrogen). The transfected cells were harvested for proliferation assay, apoptosis assay and mRNA expression after 48-hours of transfection.

\section{Apoptosis assay}

The apoptotic cells were evaluated with flow cytometry assay using FITC Annexin V Apoptosis Detection Kit with PI (BioLegend, 640914) in accordance with the manufacturer's instructions. Briefly, HaCaT was seeded at $6 \times 10^{4}$ cells per well in a 12-well plate overnight. 48-hours after transfection, culture medium containing transfected solution was replaced with complete medium for 24 hours before analysis. The transfected cells were trypsinized and washed with PBS buffer twice. Next, the cells were stained with FITC Annexin $\mathrm{V}$ and Propidium Iodide (PI) for 15 minutes in the dark at room temperature. After staining, the apoptotic cells were read by BD LSR II flow cytometer (BD Biosciences) and analyzed with the FlowJo 10 cytometry analysis software (FLOWJO, Ashland, OR, USA). All experiments were independently performed in triplicate.

\section{Cell cycle assay}

For cell cycle analysis, HaCaT were trypsinized at 48 hours after transfection. The cells were then washed with PBS buffer and fixed by adding drop-size cold $70 \%$ ethanol for 4 hours at $4^{\circ} \mathrm{C}$. After fixation, the cells were washed with PBS buffer twice and treated with $50 \mu \mathrm{l}$ of a $100 \mu \mathrm{g} / \mathrm{ml}$ RNaseA. Next, the cells were stained with $200 \mu \mathrm{l}$ of a $50 \mu \mathrm{g} / \mathrm{ml}$ PI (BioLegend, 42130) and then read by BD LSR II flow cytometer. Finally, the data were analyzed using FlowJo 10 Software for cell cycle analysis. All experiments were independently performed in triplicate.

\section{Cell viability assay}

HaCaT was seeded at $3 \times 10^{4}$ cells per well in a 24 -well plate overnight. The cells were transfected with synthesis miRNA for 48 hours as described above. The transfected cells were trypsinized and seeded at $3 \times 10^{3}$ of a 96-well plate. Viable cells were evaluated with CellTiter $96^{\circ}$ AQueous One Solution Cell Proliferation Assay (MTS assay) according to the manufacturer's instructions (Promega, G3582) at 0, 1, 2, 3, 4 and 5 days. In brief, $20 \mu \mathrm{l}$ MTS solution was added into each well of a 96-well plate containing transfected cells in $100 \mu \mathrm{l}$ of culture medium and incubated for 3 hours at $37^{\circ} \mathrm{C}$ in a $5 \%$ $\mathrm{CO}_{2}$ incubator. The absorbance was measured at OD $490 \mathrm{~nm}$ using Thermo Scientific ${ }^{\text {TM }}$ Varioskan $^{\text {tw }}$ Flash Multimode Reader (Thermo Scientific). The experiment was independently performed in triplicates.

\section{Western blot analysis}

At 48 hours post transfection, HaCaT were trypsinized and washed with PBS buffer twice. Cells were lysed with RIPA lysis buffer (Merck, Germany) containing Halt ${ }^{\text {Th }}$ Protease Inhibitor Cocktail (Thermo Scientific, USA) and protein concentration was quantified BCA assay (Thermo Scientific). A total of $20 \mu \mathrm{g}(\mathrm{GAPDH})$ and $60 \mu \mathrm{g}(\mathrm{CASP} 3)$ proteins were separately loaded onto $12 \%$ SDS-PAGE gels and were transferred onto a polyvinylidene difluoride membrane (PVDF; GE Healthcare, UK). The membrane proteins were immunoblotted with primary mAb against Cleaved Caspase-3 (dilution, 1:1000; (cat\#9661); Cell Signaling Technologies, USA) and GAPDH mouse monoclonal IgG1 (dilution, 1:5000; (cat\#sc-47724); Santa Cruz Biotechnology, USA). After washing, the protein membrane of anti-GAPDH was incubated with anti-mouse horseradish peroxidase-conjugated secondary antibody (1:5000; (cat\#sc-516102); Santa Cruz Biotechnology), while the membrane of anti-CASP3 was incubated with anti-rabbit horseradish peroxidase-conjugated secondary antibody (1:5000; (cat\#sc-2030); Santa Cruz Biotechnology). The signals were analyzed with enhanced SuperSignal ${ }^{\text {Th }}$ West Femto Maximum Sensitivity Substrate (Thermo Scientific). Images were quantified using a Licor-odyssey.

\section{Statistical analysis}

All assays were performed on at least 3 independent experiments and the results are presented as mean \pm SEM. All $p$-values were calculated by a two-tailed Student's $t$-test using IBM SPSS statistic version 22 software, $p$-values $<0.05$ were considered significant.

\section{Results}

\section{Expression of miRNA in Psoriatic skin lesion before and after treatment.}

Initially, we examined the expression of miR-155, miR$135 \mathrm{~b}$ and miR-125b in normal skin from healthy individuals compared to psoriatic skin lesion. The levels of miR-155 and miR-135b expression were significantly increased, while miR$125 \mathrm{~b}$ expression was non-significantly reduced in psoriatic skin lesion (Figure 1A, 1B, 1C). Furthermore, 7 paired-psoriatic skin lesions before and after treatment with MTX and 4 paired-psoriatic skin lesions before and after treatment with NB-UVB were analyzed. Interestingly, the level of miR-155 expression was significantly reduced after treatment in both groups treated with MTX $(P<0.01)$ or NB-UVB $(P<0.05)$ (Figure 1A), while levels of miR-135b was significantly decreased only after treatment with $\operatorname{MTX}(P<0.01)$, but not NB-UVB (Figure 1B). 


\section{(A) miR-155}
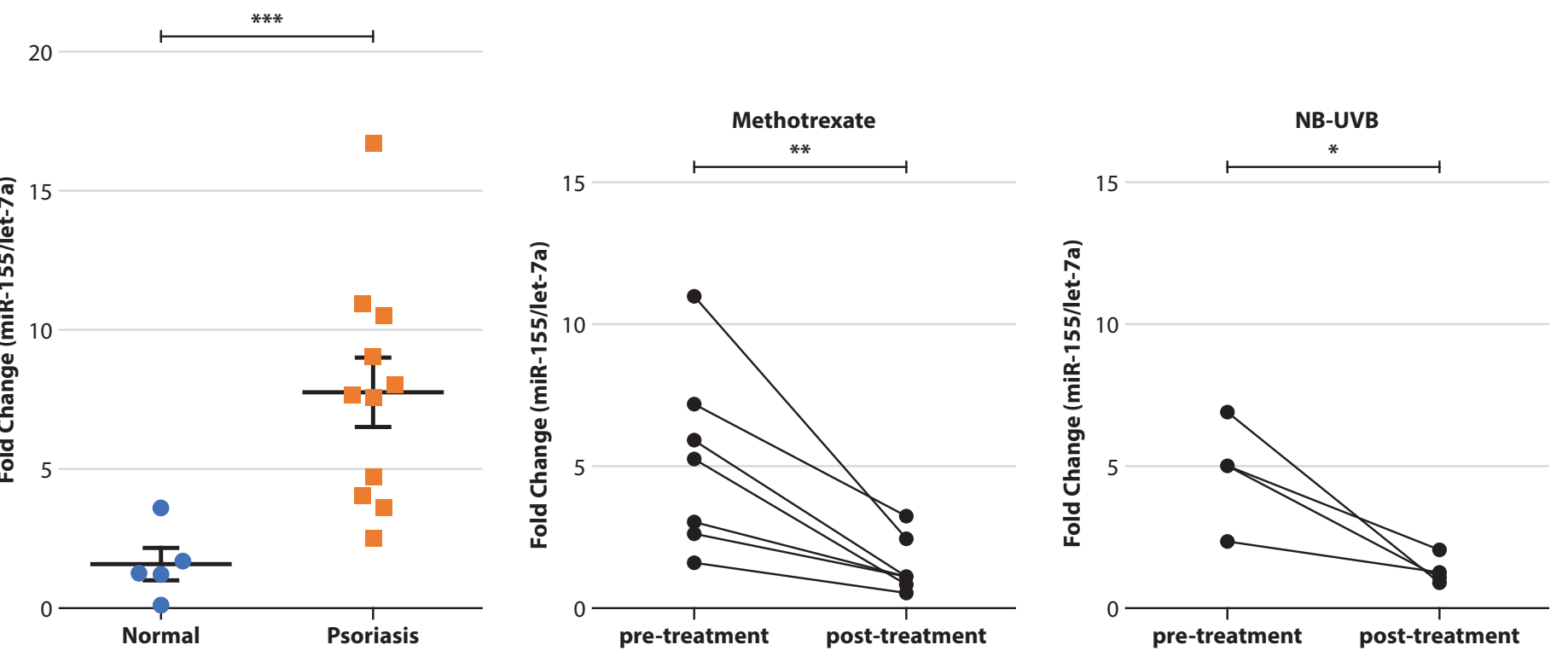

\section{(B) miR-135b}
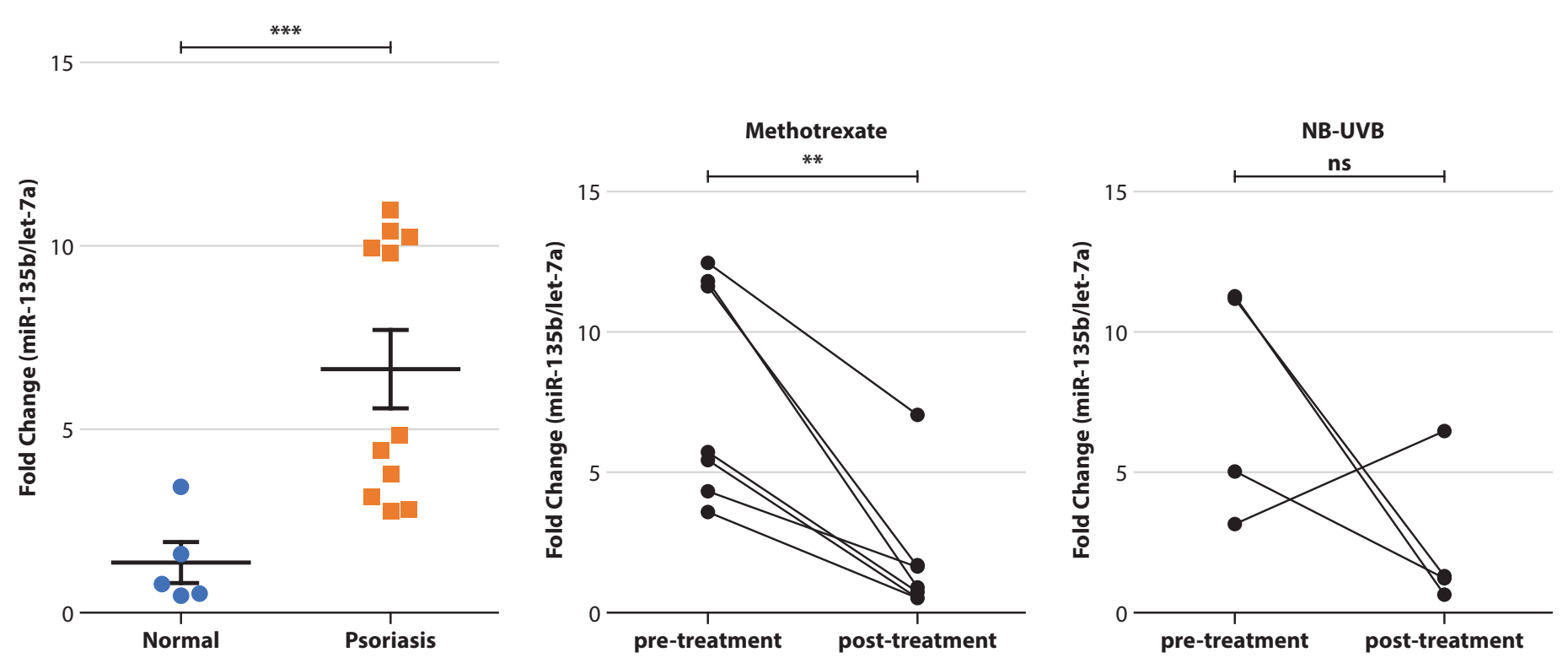

Figure 1. The expression level of miR-155 (A), miR-135b (B) and miR-125b (C) were compared between psoriasis $(\mathrm{N}=11)$ and healthy control $(\mathrm{N}=5)$ using unpaired $t$-test. In addition, 7 paired-psoriatic skin lesions before and after treatment with MTX and 4 paired-psoriatic skin lesions before and after treatment with NB-UVB were analyzed using paired $t$-test. Values are mean \pm SEM, ${ }^{*}$ indicates $P<0.01$, ${ }^{*}$ indicates $P<0.05$ and $n$ indicates non-significant. 


\section{(C) $m i R-125 b$}
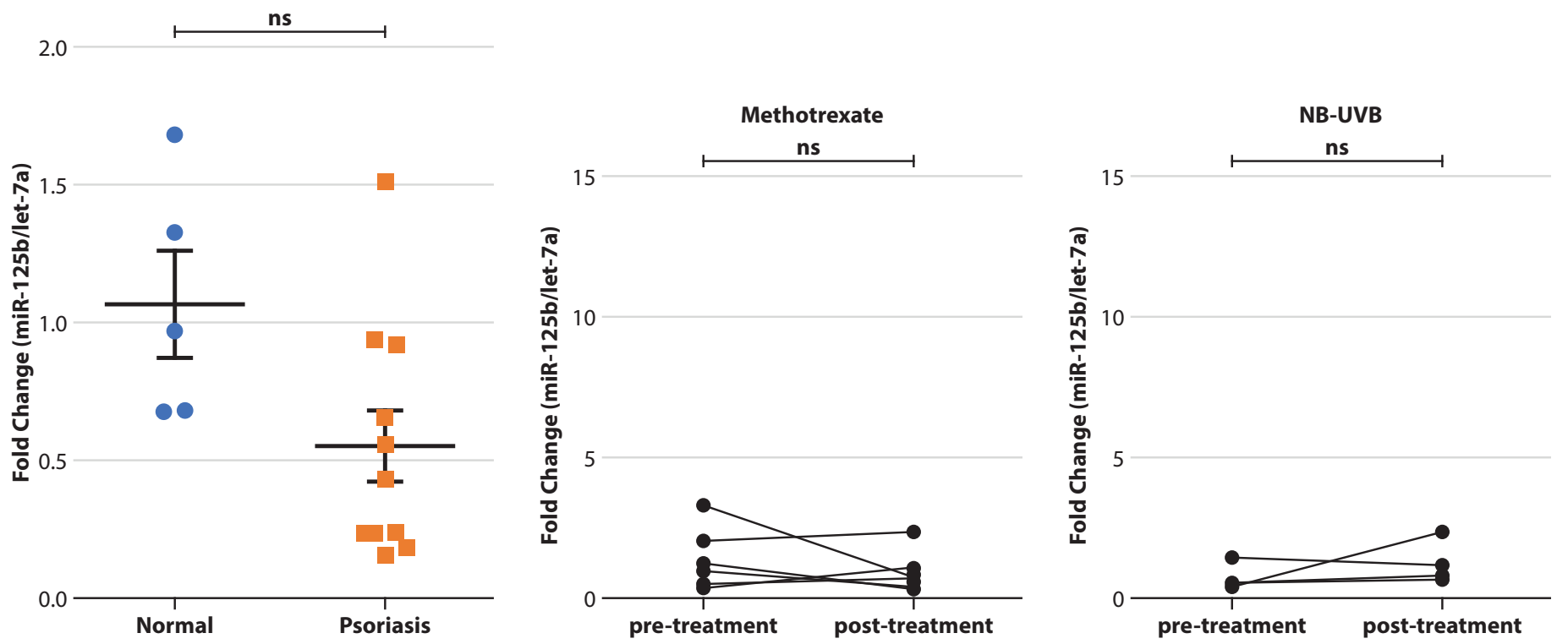

Figure 1. (Continued)

MiR-155 inhibits apoptosis of keratinocyte and increases cell viability.

In order to explore the role of miR-155 on apoptosis of keratinocyte, HaCaTs were transfected with the miR-155 mimic, and transfected cells were analyzed for annexin V-FITC/ PI staining by flow cytometry. A significant reduction of apoptotic cells (early and late apoptotic cells) was observed in transfected cells with miR-155 mimic $(5.72 \pm 0.47)$ in comparison with control mimic $(8.59 \pm 0.20)$ (Figure 2A). Subsequently, cytometric analysis was performed to determine effect of miR-155 on cell cycle. Overexpression of miR-155 increased cell population in G0/G1 phase and significantly with concomitant lost from S phase compared with control mimic (mimic: $\mathrm{G} 0 / \mathrm{G} 1=34.93 \pm 0.43 \%$ and $\mathrm{S}=45.30 \pm 0.31 \%$ vs. control: $\mathrm{G} 0 / \mathrm{G} 1=32.00 \pm 0.61 \%$ and $\mathrm{S}=48.17 \pm 0.63 \%$ ), (Figure 2B). These results revealed that miR-155 induced HaCaT cell cycle arrest at G0/G1 phase. Furthermore, cell viability was assessed using the MTS assay, which the absorbance at OD 490 was based on the number of viable cells. We found that overexpression of miR-155 mimic significantly increased cell viability of keratinocytes (Figure 2C). The transfection efficiency was determined by RT-qPCR. Results show significantly higher levels of expression of miR-155 in the transfectants when compared to the negative control (Figure 2D).
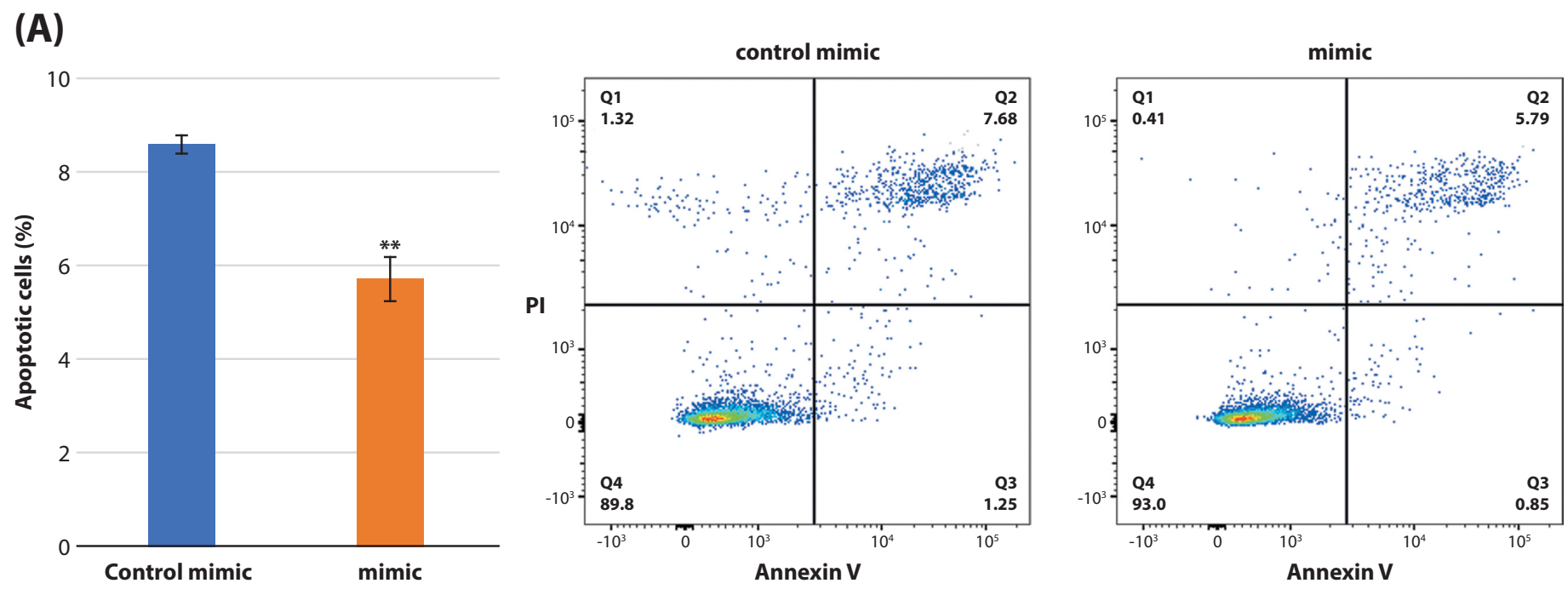

Figure 2. The analysis of effect of miR-155 on apoptosis and cell cycle of HaCaT. (A) The percentage of apoptotic keratinocytes (Q2 and Q3) after transfection with miR-155 at 24 hours. (B) The percentage of cell cycle phase in HaCaT cell after transfected with miR-155 for $48 \mathrm{~h}$. (C) The rate of viable cell of keratinocyte after transfection with miR-155 at $0,1,2,3,4$ and 5 days (D) Transiently transfected efficiency of miR-155 in HaCaT. Values are mean \pm SEM from 3 replicates. Experiment was performed 3 times independently. ${ }^{\star}$ indicates $P<0.05$, ${ }^{\star *}$ indicates $P<0.01$ and ${ }^{\star * *}$ indicates $P<0.001$. 
(B)

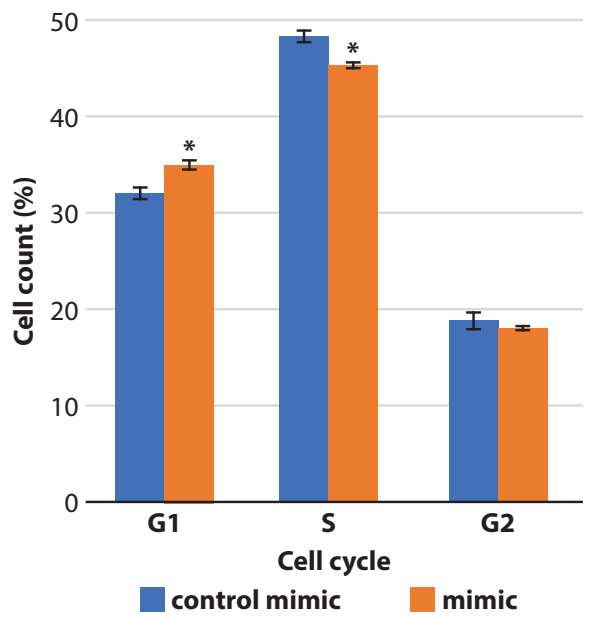

(C)

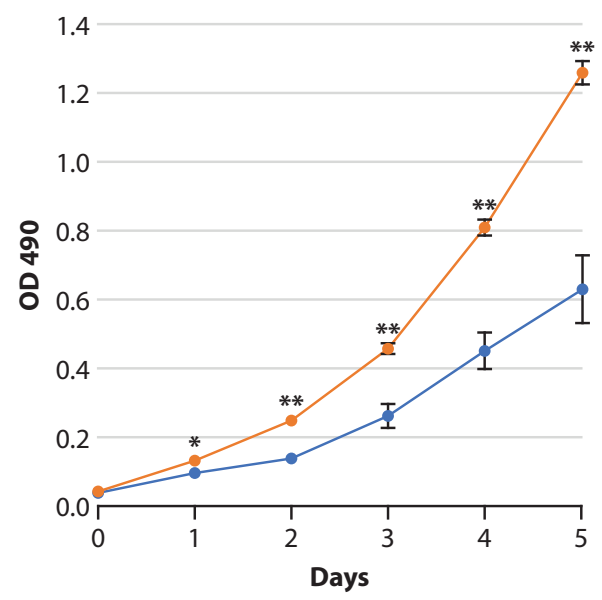

- control mimic $-\mathrm{O}$ mimic
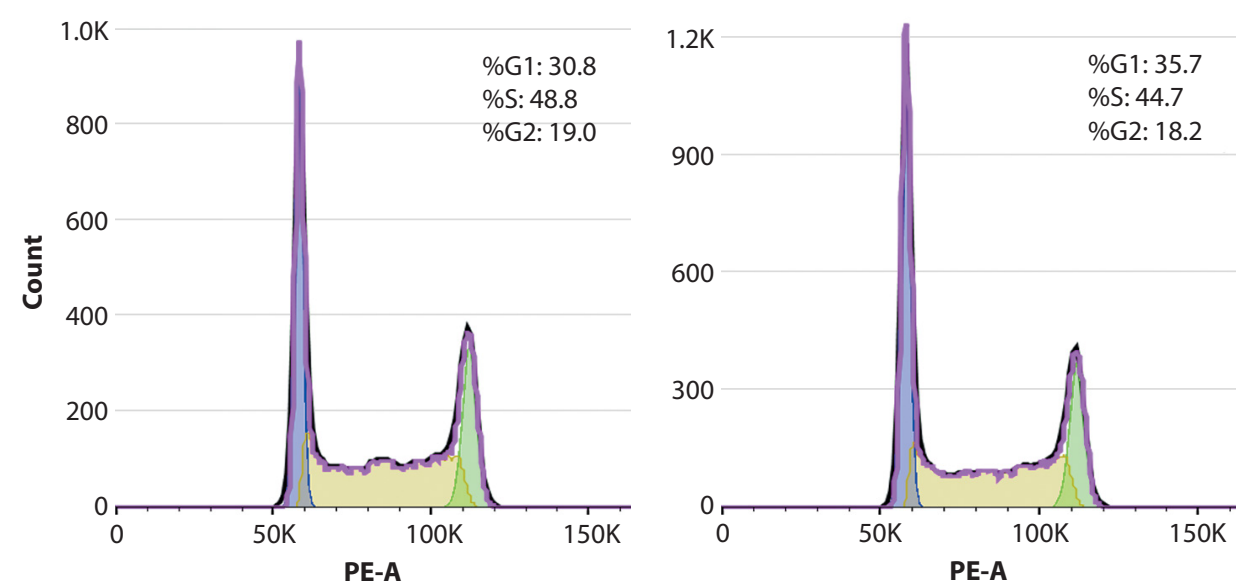

(D)

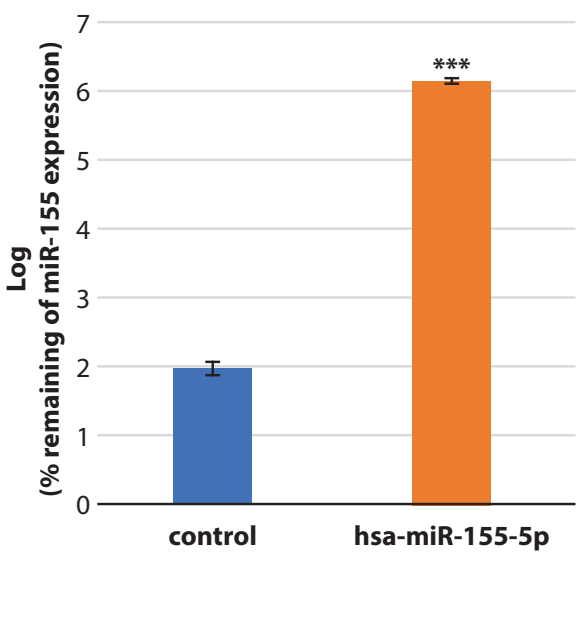

Figure 2. (Continued)

(A)
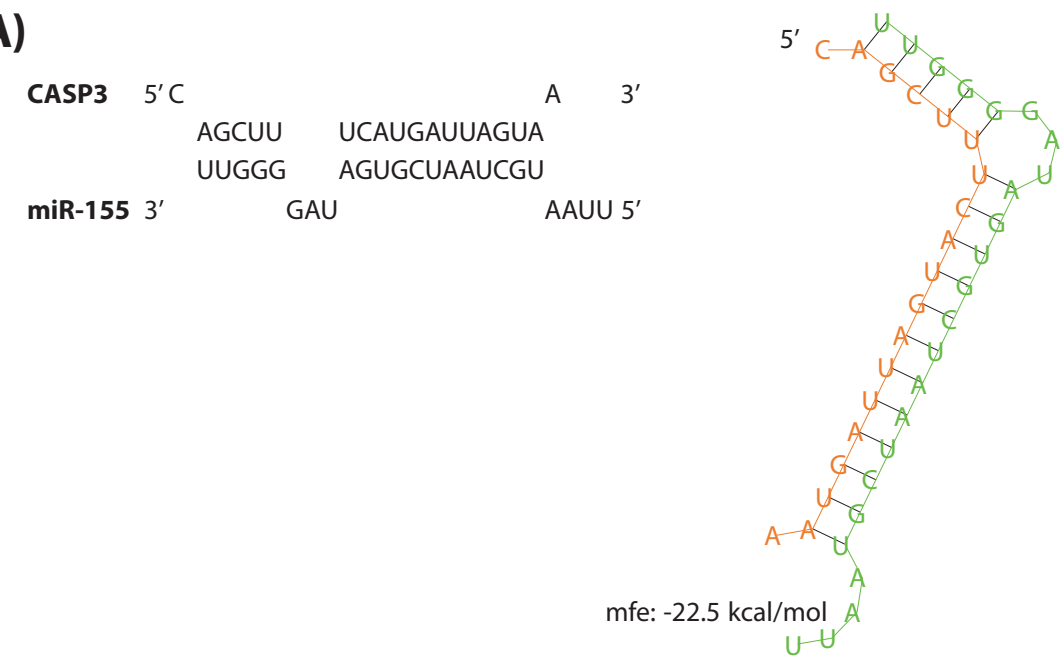

Figure 3. miR-155 mediated apoptotic suppression in HaCaT via reduce of caspase-3. (A) The position on 3'UTR CASP3 gene which is the target for miR-155. (B) The mRNA expression of CASP3 in miR-155 transfected HaCaT at $48 \mathrm{~h}$. (C) The protein expression of CASP3 in miR-155 transfectants at $48 \mathrm{~h}$. Values are mean \pm SEM for 3 replicates. Experiment was performed 3 times independently. ${ }^{\star *}$ indicates $P<0.001$. 
(B)

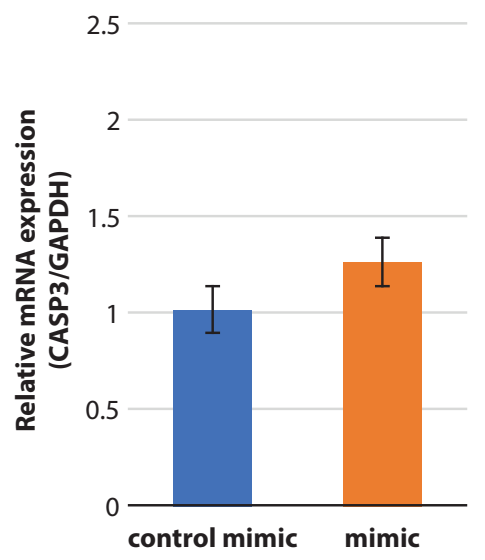

(C)

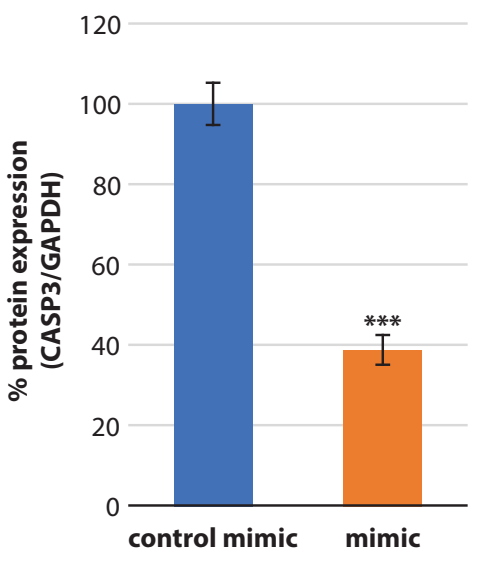

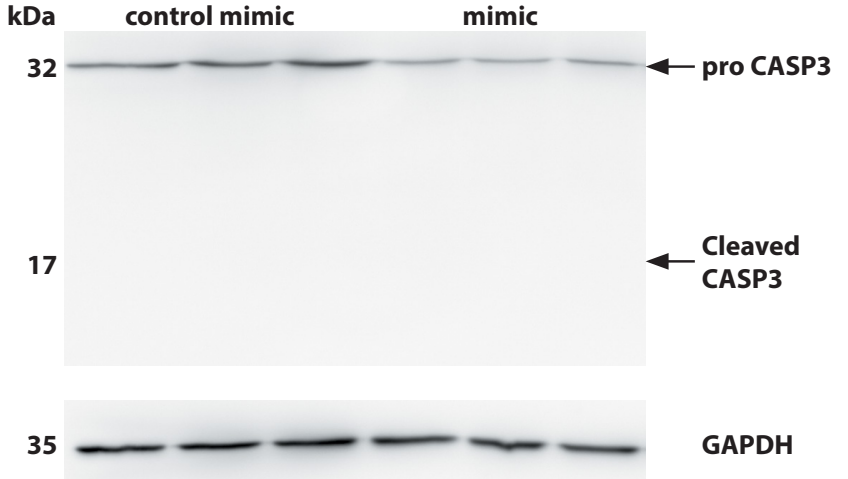

Figure 3. (Continued)

Overexpression of miR-155 suppress apoptotic activity correlated with caspase- 3

Since miRNA targets to 3'-untranslated regions (3'-UTR) of mRNA, miRWalk $3^{22}$ and RNAhybrid ${ }^{23}$ database online were utilized for searching the possible target mRNA for miR155 to understand the possible mechanisms that might underlie miR-155-mediated apoptotic suppression. Furthermore, previous study reported that miR-155 target to the 3'-UTR of $h C A S P 3$ mRNA by luciferase reporter assay. ${ }^{24,25}$ Therefore, we identified the caspase-3 (CASP3) gene as a target of miR-155 in $\mathrm{HaCaT}$ cell (Figure 3A). In addition, miR-155 was overexpressed in $\mathrm{HaCaT}$ to detect the expression level of CASP3 gene and protein by qRT-PCR and western blot. CASP3 protein was significantly decreased in the miR-155-overexpression group (Figure 3C), while mRNA expression of CASP3 was not significantly different (Figure $3 \mathbf{B}$ ). Therefore, CASP3 is one target gene of miR-155 in keratinocyte.

\section{Discussion}

The main clinical characteristics of psoriatic skin lesion are erythematous plaque cover with silvery scale which is a consequence of abnormal keratinocyte proliferation, apoptosis and terminal differentiation. ${ }^{1}$ miRNAs are known to have a key role in cell proliferation, differentiation, apoptosis, signal transduction and organ development. ${ }^{7}$ Several publications revealed the alteration of miRNAs in psoriasis. ${ }^{9}$ miR-155 is known for its functional role in various immune cells, i.e. B cells, macrophages, dendritic cells, $\mathrm{T}$ cells and regulatory $\mathrm{T}$ cells. ${ }^{26}$ In this study, we confirmed the significant increase of miR-155 in psoriatic skin lesion and illustrated the significant diminution after treatment with either MTX or NB-UVB. In addition, we identified the role of miR-155 on psoriasis by focusing on a keratinocyte cell line, HaCaT.

Nowadays, MTX and NB-UVB are still widely used as an effective conventional treatment for psoriasis. ${ }^{27,28}$ We determined the expression of miR-155, miR-125b and miR-135b on psoriatic skin lesions before and after both conventional treatments. Both treatments could suppress miR-155 expression in psoriatic skin lesions in all patients, while only MTX could reduce miR-135b expression. This finding supports the relevance of miR-155 in the pathogenesis of psoriasis. Previous reports demonstrated that both MTX and NB-UVB could suppress proliferation and induce apoptosis of $\mathrm{T}$ lymphocytes and keratinocytes in psoriasis. ${ }^{29,30}$ Therefore, it is suspected that the effective treatment of MTX and NB-UVB in psoriasis may be a result of down-regulation of miR-155 especially by inducing apoptosis of keratinocytes.

The results of our study revealed that overexpression of miR-155 could reduce apoptosis, induce cell cycle arrest at G0/G1 phase, promote viable HaCaT but not induce cell proliferation. Previous studies revealed that miR-155 reduced apoptosis by targeting 3'UTR CASP3 gene and inhibits apoptosis activity of macrophages and nucleus pulposus. ${ }^{24,25}$ These evidences suggested that miR-155 may be involved in psoriatic skin lesion by decreasing apoptosis and maintaining cell viability of keratinocytes.

Previous publications reported up-regulation of miR-135b and down-regulation of miR-125b in psoriatic skin. ${ }^{13,31,32}$ Our study demonstrated significant up-regulation of miR-135b in psoriatic skin lesion which is corresponded with publication. However, our study could neither reveal significant change of miR-125b in psoriatic skin nor significant change of miR-135 after treatment with NB-UVB. These insignificances might be due to the small number of samples.

Apoptosis is known as keratinocyte regulator in maintenance of epidermal homeostasis. Our results show that overexpressed miR-155 inhibits HaCaT apoptosis and reduces the expression of CASP3 protein. Increasing caspase- 3 activity is one target of psoriasis treatment. Previous data indicated that MTX induces apoptosis in psoriatic skin biopsy by increasing apoptotic marker such as caspase-3 and caspase-9, and decreasing anti-apoptosis marker $(\mathrm{Bcl}-\mathrm{xL}){ }^{33} \mathrm{NB}-\mathrm{UVB}$ treatment is also an induction of apoptotic activity on psoriatic keratinocyte to allow resolution malfunctioned apoptotic mechanism and clearance of psoriatic keratinocytes. ${ }^{34}$ Thus, it is possible that the psoriatic keratinocyte resisting to apoptosis might be through up-regulation of miR-155 by decreasing caspase-3 activity. Besides, apoptotic effect of MTX and NBUVB by increasing caspase- 3 could be due to down-regulation of miRNA-155. 


\section{Conclusion}

In conclusion, this study displays the effect of MTX and NB-UVB on miR-155 in psoriatic skin lesions. Additionally, the functional roles of miR-155 on keratinocytes have been exhibited. miR-155 displays a role of reducing apoptotic HaCaT by down-regulating CASP3 protein. This finding leads to a more understanding in the pathogenesis of psoriasis and might be a clue to develop a new diagnostic or therapeutic modality for psoriasis in the near future.

\section{Acknowledgments}

This work was supported by a research grant from the Government Research Budget (2016), Thailand Research Fund (TRG 5480010) and Ratchadapisek Somphot Fund for Postdoctoral Fellowship, Chulalongkorn University.

\section{References}

1. Nestle FO, Kaplan DH, Barker J. Mechanisms of disease: psoriasis. New Engl J Med. 2009;361:496-509.

2. Ruchusatsawat K, Wongpiyabovorn J, Protjaroen P, Chaipipat $M$, Shuangshoti S, Thorner PS, et al. Parakeratosis in skin is associated with loss of inhibitor of differentiation 4 via promoter methylation. Hum Pathol. 2011;42(12):1878-87.

3. Montaudié H, Sbidian E, Paul C, Maza A, Gallini A, Aractingi S, et al. Methotrexate in psoriasis: a systematic review of treatment modalities, incidence, risk factors and monitoring of liver toxicity. J Eur Acad Dermatol Venereol. 2011;25(s2):12-8.

4. Fagerli KM, Lie E, van der Heijde D, Heiberg MS, Lexberg ÅS, Rødevand E, et al. The role of methotrexate co-medication in TNF-inhibitor treatment in patients with psoriatic arthritis: results from 440 patients included in the NOR-DMARD study. Ann Rheum Dis. 2014;73(1):132-7.

5. Bartel DP. MicroRNAs: genomics, biogenesis, mechanism, and function. Cell. 2004;116(2):281-97.

6. He L, Hannon GJ. MicroRNAs: small RNAs with a big role in gene regulation. Nature Rev Genet. 2004;5:522.

7. Løvendorf MB, Mitsui H, Zibert JR, Røpke MA, Hafner M, Dyring-Andersen B, et al. Laser capture microdissection followed by next-generation sequencing identifies disease-related microRNAs in psoriatic skin that reflect systemic microRNA changes in psoriasis. Exp Dermatol. 2015;24(3):187-93.

8. Yan S, Xu Z, Lou F, Zhang L, Ke F, Bai J, et al. NF- $\kappa$ B-induced microRNA-31 promotes epidermal hyperplasia by repressing protein phosphatase 6 in psoriasis. Nat Commun. 2015;6:7652.

9. Ichihara A, Jinnin M, Yamane K, Fujisawa A, Sakai K, Masuguchi S, et al. microRNA-mediated keratinocyte hyperproliferation in psoriasis vulgaris. Br J Dermatol. 2011;165(5):1003-10.

10. Xia P, Fang X, Zhang ZH, Huang Q, Yan KX, Kang KF, et al. Dysregulation of miRNA146a versus IRAK1 induces IL-17 persistence in the psoriatic skin lesions. Immunol Lett. 2012;148(2):151-62.

11. Bucci M, Ianni U, Celletti E, Sabatini E, De Cesare D, Cipollone F. Role of microRNA-155 in the pathogenesis and treatment of psoriatic arthritis. Nutr Metab Cardiovasc. 2017;27(1):e10-1.

12. Lerman G, Avivi C, Mardoukh C, Barzilai A, Tessone A, Gradus B, et al. MiRNA expression in psoriatic skin: reciprocal regulation of hsa-miR-99a and IGF-1R. Plos One. 2011;6(6):e20916.

13. Xu N, Brodin P, Wei T, Meisgen F, Eidsmo L, Nagy N, et al. MiR-125b, a microRNA downregulated in psoriasis, modulates keratinocyte proliferation by targeting FGFR2. J Invest Dermatol. 2011;131(7):1521-9.
14. Yu X, An J, Hua Y, Li Z, Yan N, Fan W, et al. MicroRNA-194 regulates keratinocyte proliferation and differentiation by targeting Grainyhead-like 2 in psoriasis. Pathol Res Pract. 2017;213(2):89-97.

15. Zhu H, Hou L, Liu J, Li Z. MiR-217 is down-regulated in psoriasis and promotes keratinocyte differentiation via targeting GRHL2. Biochem Biophys Res Commun. 2016;471(1):169-76.

16. Bertoli G, Cava C, Castiglioni I. MicroRNAs: new biomarkers for diagnosis, prognosis, therapy prediction and therapeutic tools for breast cancer. Theranostics. 2015;5(10):1122.

17. Kurowska-Stolarska M, Alivernini S, Ballantine LE, Asquith DL, Millar NL, Gilchrist DS, et al. MicroRNA-155 as a proinflammatory regulator in clinical and experimental arthritis. PNAS. 2011;108(27):11193-8.

18. O'Connell RM, Kahn D, Gibson WSJ, Round JL, Scholz RL, Chaudhuri AA, et al. MicroRNA-155 promotes autoimmune inflammation by enhancing inflammatory T cell development. Immunity. 2010;33(4):607-19.

19. Rodriguez A, Vigorito E, Clare S, Warren MV, Couttet P, Soond DR, et al Requirement of bic/microRNA-155 for normal immune function. Science. 2007;316(5824):608-11.

20. Boss IW, Nadeau PE, Abbott JR, Yang Y, Mergia A, Renn R. A Kaposi's sarcoma-associated herpesvirus-encoded ortholog of MicroRNA miR-155 induces human splenic B-Cell expansion in NOD/LtSz-scid IL2R $\gamma($ null $)$ mice. J Virol. 2011;85(19):9877-86.

21. Schmittgen TD, Livak KJ. Analyzing real-time PCR data by the comparative CT method. Nat Protoc. 2008;3:1101.

22. Sticht C, De La Torre C, Parveen A, Gretz N. miRWalk: An online resource for prediction of microRNA binding sites. Plos One. 2018;13(10):e0206239.

23. Rehmsmeier M, Steffen P, Höchsmann M, Giegerich R. Fast and effective prediction of microRNA/target duplexes. RNA. 2004;10(10):1507-17.

24. Wang HQ, Yu XD, Liu ZH, Cheng X, Samartzis D, Jia LT, et al. Deregulated miR-155 promotes Fas-mediated apoptosis in human intervertebral disc degeneration by targeting FADD and caspase-3. J Pathol. 2011;225(2): 232-42.

25. De Santis R, Liepelt A, Mossanen JC, Dueck A, Simons N, Mohs A, et al miR-155 targets Caspase-3 mRNA in activated macrophages. RNA Biol. 2016;13(1):43-58.

26. Lind EF, Ohashi PS. Mir-155, a central modulator of T-cell responses. Eur J Immunol. 2014;44(1):11-5.

27. Kumar B, Saraswat A, Kaur I. Short-term methotrexate therapy in psoriasis: a study of 197 patients. Int J Dermatol. 2002;41(7):444-8.

28. Dogra S, Mahajan R. Systemic methotrexate therapy for psoriasis: past, present and future. Clin Exp Dermatol. 2013;38(6):573-88.

29. Piskin G, Tursen U, Sylva-Steenland RMR, Bos JD, Teunissen MBM Clinical improvement in chronic plaque-type psoriasis lesions after narrow-band UVB therapy is accompanied by a decrease in the expression of IFN- $\gamma$ inducers - IL-12, IL-18 and IL-23. Exp Dermatol. 2004;13(12): 764-72.

30. Kleinpenning MM, Smits T, Boezeman J, Van De Kerkhof PCM, Evers AWM, Gerritsen MJP. Narrowband ultraviolet B therapy in psoriasis: randomized double-blind comparison of high-dose and low-dose irradiation regimens. Br J Dermatol. 2009;161(6):1351-6.

31. Gu X, Nylander E, Coates PJ, Nylander K. Effect of narrow-band ultraviolet B phototherapy on 663 and microRNA (miR-21 and miR-125b) expression in psoriatic epidermis. Acta Derm Venereol. 2011;91(4):392-7.

32. Joyce CE, Zhou X, Xia J, Ryan C, Thrash B, Menter A, et al. Deep sequencing of small RNAs from human skin reveals major alterations in the psoriasis miRNAome. Hum Mol Gen. 2011;20(20):4025-40.

33. Elango T, Thirupathi A, Subramanian S, Ethiraj P, Dayalan H, Gnanaraj P. Methotrexate treatment provokes apoptosis of proliferating keratinocyte in psoriasis patients. Int J Clin Exp Med. 2017;17(3):371-81.

34. Weatherhead SC, Farr PM, Jamieson D, Hallinan JS, Lloyd JJ, Wipat A, et al. Keratinocyte apoptosis in epidermal remodeling and clearance of psoriasis induced by UV radiation. J Invest Dermatol. 2011;131(9):1916-26. 